\title{
Prevalence of thyroid autoimmunity and subclinical hypothyroidism in persons with chronic kidney disease not requiring chronic dialysis
}

\author{
Giovanni Targher ${ }^{1, *}$, Michel Chonchol², \\ Giacomo Zoppini ${ }^{1}$, Gianluca Salvagno ${ }^{3}$, Isabella \\ Pichiri ${ }^{1}$, Massimo Franchini ${ }^{4}$ and Giuseppe \\ Lippi $^{3}$ \\ ${ }^{1}$ Section of Endocrinology, Department of \\ Biomedical and Surgical Sciences, University \\ Hospital of Verona, Verona, Italy \\ ${ }^{2}$ Division of Renal Diseases and Hypertension, \\ University of Colorado Denver Health Sciences \\ Center, Denver, USA \\ ${ }^{3}$ Section of Clinical Chemistry, Department of \\ Biomedical and Morphological Sciences, University \\ Hospital of Verona, Verona, Italy \\ ${ }^{4}$ Immuno-Hematology and Transfusion Center, \\ Department of Pathology and Laboratory Medicine, \\ University Hospital of Parma, Parma, Italy
}

\begin{abstract}
Background: The prevalence of thyroid autoimmunity and subclinical primary hypothyroidism in persons with chronic kidney disease (CKD) not requiring chronic dialysis is not well defined.

Methods: We studied 1000 consecutive adult outpatients who were referred by their general practitioner for blood testing over the last 2 years. We excluded those with abnormal serum free thyroxine (FT4) levels $(n=85)$. No participants required chronic renal replacement therapy. Thyroid autoimmunity was defined as increased concentrations of serum anti-thyroid antibodies. Subclinical primary hypothyroidism was defined as a serum thyrotropin (TSH) concentration $>4 \mathrm{mIU} / \mathrm{L}$. CKD was defined as an estimated glomerular filtration rate (eGFR) $<60 \mathrm{~mL} / \mathrm{min} / 1.73 \mathrm{~m}^{2}$.

Results: Overall, 53 (5.8\%) subjects had eGFR $<60 \mathrm{~mL} / \mathrm{min} / 1.73 \mathrm{~m}^{2}$. Of these, $98(10.7 \%)$ had subclinical hypothyroidism, and $213(23.3 \%)$ subjects had increased anti-thyroid antibodies. Approximately 26\% and $34 \%$ of those with eGFR $<60 \mathrm{~mL} / \mathrm{min} / 1.73 \mathrm{~m}^{2}$ had laboratory evidence of subclinical hypothyroidism or thyroid autoimmunity, respectively. In subgroup analysis stratified by TSH and thyroid autoimmunity, decreasing eGFR values appeared to be more strongly related with the increase in TSH rather than antithyroid antibodies.
\end{abstract}

*Corresponding author: Dr. Giovanni Targher, Section of Endocrinology, Department of Biomedical and Surgical Sciences, University of Verona, Ospedale Civile Maggiore, Piazzale Stefani, 1, 37126 Verona, Italy Phone: +39-045-8123748, Fax: +39-045-8027314, E-mail: giovanni.targher@univr.it Received June 4, 2009; accepted July 17, 2009; previously published online September 22, 2009
Conclusions: Thyroid autoimmunity and subclinical primary hypothyroidism are highly prevalent in persons with CKD not requiring chronic dialysis. Clin Chem Lab Med 2009;47:1367-71.

Keywords: epidemiology; estimated glomerular filtration rate; subclinical hypothyroidism; thyroid autoimmunity; thyroid dysfunction.

\section{Introduction}

There is extensive linkage between the kidneys and thyroid function in the embryonic stage. This linkage becomes more evident in clinically overt hyperthyroidism and hypothyroidism. These conditions lead to hemodynamic and vascular changes in the kidney, as well as to changes in the structure and function of the tubules and glomerulus (1). An increased prevalence of goiter and thyroid gland volume has been reported in patients with chronic kidney disease (CKD) $(2,3)$. It has been suggested that clinically overt hypothyroidism may be more common in patients with CKD compared with the general population (1).

Interestingly, CKD was found to be associated with a greater prevalence of clinical and subclinical primary hypothyroidism among participants of the Third National Health and Nutrition Examination Survey (4). Recently, we extended these findings in a large nonUS cohort of outpatients by showing that the prevalence of subclinical hypothyroidism increases as estimated glomerular filtration rate (eGFR) decreases (5). These findings may have important clinical implications because subclinical primary hypothyroidism, independent of its specific etiology, is associated with an increased risk of cardiovascular events (6-8).

At present, there is no information about the prevalence of subclinical primary hypothyroidism caused by chronic autoimmune thyroiditis, the most common cause of primary hypothyroidism (9), in persons with CKD who do not require maintenance dialysis.

The purpose of this study was to investigate the prevalence of thyroid autoimmunity and subclinical primary hypothyroidism at different levels of kidney function in a large sample of unselected adult outpatients.

\section{Materials and methods}

A cross-sectional analysis of the database of the Laboratory Information System of the Clinical Chemistry Laboratory at the Verona University Hospital was performed. We retrieved serum creatinine and thyroid function test results from all 
adult outpatients, 18 years of age or older $(n=1000)$, who were consecutively referred for testing by general practitioners over the last 2 years (from April 2007 to April 2009). If a subject had more than one blood test ordered over the 2 years, only the first result was included in the analysis. For this study, participants who had abnormal serum free thyroxine (FT4) concentrations $(n=85)$ were excluded. No participants required chronic renal replacement therapy. Thus, the final sample used in this study included 915 outpatient adults. The local Ethics Committee approved the study protocol.

Venous blood was collected from all participants in the morning following an overnight fast. Serum glucose and lipids were measured using enzymatic procedures with a Roche/Hitachi Modular System P (Roche Diagnostics GmbH, Milan, Italy), according to the manufacturer's specifications and employing proprietary reagents. Low-density lipoprotein (LDL)-cholesterol was calculated with the Friedewald formula. Serum creatinine was measured using a Roche/Hitachi Modular System $\mathrm{P}$ with a Jaffè rate blanked and compensated assay. The calibrators for the Roche method were traceable to an isotope dilution mass spectrometric method for serum creatinine using standard reference methods. Serum thyrotropin (TSH) concentrations were measured with a third generation immunometric assay (functional sensitivity of $0.01-0.02 \mathrm{mIU} / \mathrm{L}$ with an inter-assay imprecision of $\sim 20 \%$ ) using the IMMULITE-2000 analyzer (Diagnostic Products Corporation, Los Angeles, CA, USA). Serum concentrations of FT4 (by a solid-phase, chemiluminescent, competitive analog immunoassay with a total imprecision of $<9.0 \%$ ), anti-thyroglobulin (anti-TG) and anti-thyroperoxidase (anti-TPO) antibodies (total imprecision of $<5.6 \%$ and $<7.0 \%$ for anti-TG and anti-TPO, respectively) were also measured with the IMMULITE-2000 analyzer. The reference range for TSH was $0.35-4.0 \mathrm{mIU} / \mathrm{L}$, for FT4 10-24 pmol/L, for anti-TG antibody $<40 \mathrm{IU} / \mathrm{mL}$ and for anti-TPO antibody $<35 \mathrm{lU} / \mathrm{mL}$. Subclinical primary hypothyroidism was defined as a serum TSH concentrations $>4.0 \mathrm{mIU} / \mathrm{L}$ with normal FT4 values. Thyroid autoimmunity was defined as increased titers of serum anti-TPO ( $>35 \mathrm{IU} / \mathrm{mL}$ ) or anti-TG ( $>40 \mathrm{IU} / \mathrm{mL})$ antibodies.

eGFR was estimated using the abbreviated modification of diet in renal disease (MDRD) equation (10) as follows: eGFR $=175 \times\left(\right.$ serum creatinine $\left.{ }^{-1.154}\right) \times\left(\right.$ age $\left.^{-0.203}\right) \times 1.212$ (if black) $\times 0.742$ (if female). For this study, CKD was defined as an eGFR $<60 \mathrm{~mL} / \mathrm{min} / 1.73 \mathrm{~m}^{2}(10)$.

\section{Statistical analysis}

Results are expressed as means \pm SD or proportions. Participants were stratified into three categories based on the eGFR (i.e., $<60,60-89$ and $\geq 90 \mathrm{~mL} / \mathrm{min} / 1.73 \mathrm{~m}^{2}$ ). In these analyses, we combined subjects with eGFR $<45 \mathrm{~mL} / \mathrm{min} /$ $1.73 \mathrm{~m}^{2}$ and those with eGFR $45-60 \mathrm{~mL} / \mathrm{min} / 1.73 \mathrm{~m}^{2}$ into a single eGFR category due to the low number of individuals with eGFR $<45 \mathrm{~mL} / \mathrm{min} / 1.73 \mathrm{~m}^{2}(\mathrm{n}=16)$. Skewed variables (triglycerides, TSH, anti-TG and anti-TPO) were logarithmically transformed to achieve normality prior to analysis. Statistical analyses included the one-way analysis of variance (for continuous variables) and the $\chi^{2}$-test with Yates's correction for continuity (for categorical measures). The joint effects of an increased TSH concentration and thyroid autoimmunity on eGFR values (included as a continuous measure) were tested using a 2-factor analysis of variance. Moreover, the independence of associations between thyroid function test results and eGFR was tested by analysis of covariance or multivariate logistic regression analysis. Adjusting variables were age, gender, serum fasting glucose and lipids. p-Values $<0.05$ were considered statistically significant.

\section{Results}

After excluding subjects $(n=85)$ with abnormal serum FT4 concentrations [i.e., those with FT4 below $10 \mathrm{pmol} / \mathrm{L}(\mathrm{n}=18)$ or above $24 \mathrm{pmol} / \mathrm{L}(\mathrm{n}=67)]$, cumulative results for main demographic variables, serum creatinine and thyroid function tests were retrieved for 915 adult outpatients with a mean age of $48.9 \pm 15$ years. There were 602 women and 313 men. The mean eGFR was $89.6 \pm 22 \mathrm{~mL} / \mathrm{min} / 1.73 \mathrm{~m}^{2}$ (range: 31-202 $\mathrm{mL} / \mathrm{min} / 1.73 \mathrm{~m}^{2}$ ). Mean serum TSH and FT4 concentrations were $2.26 \pm 2.98 \mathrm{mlU} / \mathrm{L}$ (range: $<0.001-$ $41.7 \mathrm{mIU} / \mathrm{L}$ ) and $16.8 \pm 2.8 \mathrm{pmol} / \mathrm{L}$ (range: $10-24$ $\mathrm{pmol} / \mathrm{L})$, respectively.

Most participants ( $n=729,79.7 \%$ ) had serum TSH concentrations ranging from $0.35 \mathrm{mIU} / \mathrm{L}$ to $4.0 \mathrm{mIU} / \mathrm{L}$. $10.7 \%(n=98)$ had subclinical biochemical hypothyroidism (i.e., $\mathrm{TSH}>4.0 \mathrm{mIU} / \mathrm{L})$, and $9.6 \%(\mathrm{n}=88)$ had subclinical biochemical hyperthyroidism (i.e., TSH $<0.35 \mathrm{mlU} / \mathrm{L})$. An increased anti-TPO antibody concentration was present in 172 (18.8\%) subjects, whereas an increased anti-TG antibody concentration was present in 125 (13.7\%).

Most participants $(\mathrm{n}=862,94.2 \%)$ had an eGFR $>60 \mathrm{~mL} / \mathrm{min} / 1.73 \mathrm{~m}^{2}$, while 427 had an eGFR $\geq 90 \mathrm{~mL} /$ $\mathrm{min} / 1.73 \mathrm{~m}^{2}$. Fifty three $(5.8 \%$ of total) subjects had an eGFR $<60 \mathrm{~mL} / \mathrm{min} / 1.73 \mathrm{~m}^{2}$. Of these, 37 of had an eGFR between $45 \mathrm{~mL} / \mathrm{min} / 1.73 \mathrm{~m}^{2}$ and $59 \mathrm{~mL} / \mathrm{min} /$ $1.73 \mathrm{~m}^{2}$ and 16 had an eGFR $<45 \mathrm{~mL} / \mathrm{min} / 1.73 \mathrm{~m}^{2}$.

Baseline characteristics of the participants according to eGFR categories are shown in Table 1. Participants with lower eGFR were older, more likely to be female and had significantly higher serum concentrations of TSH and anti-thyroid antibodies compared with those showing normal or near-normal kidney function. The prevalence of participants with serum TSH concentrations $>4.0 \mathrm{mUI} / \mathrm{L}$ or with increased titers of serum anti-thyroid antibodies steadily increased among persons with progressively lower eGFR values (ranging from $7.7 \%$ to $26.4 \%$ for $\mathrm{TSH}$, from $15.4 \%$ to $28.3 \%$ for anti-TPO antibody, and from $10.2 \%$ to $26.4 \%$ for anti-TG antibody). The prevalence of increased anti-TPO and/or anti-TG antibodies ranged from $18.7 \%$ to $34.0 \%$ across eGFR categories (Table 1). Serum triglycerides tended to be higher, and high density lipoprotein (HDL)-cholesterol tended to be lower among persons with progressively lower eGFR values, but these did not reach statistical significance. Serum concentrations of FT4 and fasting glucose were not significantly different among eGFR categories. Notably, as also shown in Table 1, the significant differences in TSH and anti-thyroid antibodies, observed across eGFR categories, remained unchanged even after adjusting for age and gender. Additional adjustment for fasting plasma glucose and lipid parameters did not materially alter the results (data not shown).

Conversely, compared with participants without hypothyroidism ( $n=817$ ), those with subclinical hypo- 
Table 1 Clinical and biochemical characteristics of participants stratified according to eGFR.

\begin{tabular}{|c|c|c|c|c|c|}
\hline & $\begin{array}{l}<60 \\
\mathrm{~mL} / \mathrm{min} / 1.73 \mathrm{~m}^{2}\end{array}$ & $\begin{array}{l}60-89 \\
\mathrm{~mL} / \mathrm{min} / 1.73 \mathrm{~m}^{2}\end{array}$ & $\begin{array}{l}\geq 90 \\
\mathrm{~mL} / \mathrm{min} / 1.73 \mathrm{~m}^{2}\end{array}$ & $\begin{array}{l}\text { Unadjusted } \\
\text { p-values }\end{array}$ & $\begin{array}{l}\text { Age- and sex- } \\
\text { adjusted } \\
p^{\text {p-values }}{ }^{\text {a }}\end{array}$ \\
\hline Gender (male/female) & $10 / 43$ & $120 / 315$ & $183 / 244$ & $<0.0001$ & - \\
\hline Age, years & $66 \pm 16$ & $53 \pm 13$ & $43 \pm 14$ & $<0.0001$ & - \\
\hline $\mathrm{FT} 4, \mathrm{pmol} / \mathrm{L}$ & $16.5 \pm 3.2$ & $16.7 \pm 2.6$ & $16.8 \pm 2.8$ & 0.71 & 0.54 \\
\hline Thyrotropin, mUI/L & $3.43 \pm 4.1$ & $2.41 \pm 3.5$ & $1.94 \pm 2.1$ & $<0.001$ & $<0.001$ \\
\hline Anti-TPO antibody, IU/mL & $510 \pm 2741$ & $142 \pm 584$ & $96 \pm 402$ & 0.0024 & 0.01 \\
\hline Anti-TG antibody, IU/mL & $1462 \pm 8250$ & $130 \pm 983$ & $47 \pm 240$ & $<0.001$ & 0.01 \\
\hline Thyrotropin $>4 \mathrm{mUl} / \mathrm{L}, \%$ & $26.4 \%$ & $11.7 \%$ & $7.7 \%$ & $<0.001$ & $<0.001$ \\
\hline Anti-TPO antibodies $>35 \mathrm{IU} / \mathrm{mL}, \%$ & $28.3 \%$ & $20.9 \%$ & $15.4 \%$ & $<0.01$ & 0.02 \\
\hline Anti-TG antibodies $>40 \mathrm{IU} / \mathrm{mL}, \%$ & $26.4 \%$ & $15.4 \%$ & $10.2 \%$ & 0.0019 & 0.01 \\
\hline $\begin{array}{l}\text { Increased anti-TPO and/or anti-TG } \\
\text { antibodies, } \%\end{array}$ & $34.0 \%$ & $26.4 \%$ & $18.7 \%$ & 0.004 & 0.01 \\
\hline Fasting glucose, $\mathrm{mmol} / \mathrm{L}$ & $5.12 \pm 1.1$ & $5.11 \pm 1.0$ & $5.17 \pm 1.1$ & 0.61 & ND \\
\hline Triglycerides, mmol/L & $1.36 \pm 0.65$ & $1.32 \pm 0.65$ & $1.18 \pm 0.80$ & 0.07 & ND \\
\hline HDL-cholesterol, mmol/L & $1.50 \pm 0.41$ & $1.59 \pm 0.43$ & $1.60 \pm 0.39$ & 0.11 & ND \\
\hline LDL-cholesterol, mmol/L & $3.39 \pm 0.64$ & $3.24 \pm 0.89$ & $3.22 \pm 0.85$ & 0.72 & ND \\
\hline eGFR, $\mathrm{mL} / \mathrm{min} / 1.73 \mathrm{~m}^{2}$ & $49 \pm 9$ & $77 \pm 8$ & $107 \pm 18$ & ND & ND \\
\hline
\end{tabular}

Cohort size $=915$. Data are expressed as means \pm SD or proportions. Unadjusted p-values for trends by the one-way analysis of variance (for continuous variables) or the $\chi^{2}$-test (for categorical variables). ${ }^{a}$ Adjusted $p$-values for trends by the analysis of covariance (for continuous variables) or the logistic regression analysis (for categorical measures). eGFR, estimated glomerular filtration rate; FT4, free thyroxine; anti-TPO, anti-thyroperoxidase; anti-TG, anti-thyroglobulin; ND, not determined; HDL, high-density lipoprotein; LDL, low-density lipoprotein.

thyroidism ( $\mathrm{n}=98$ ) were slightly older, less likely to be male and had slightly higher LDL-cholesterol, and lower eGFR values $(81.1 \pm 22$ vs. $91.2 \pm 22 \mathrm{~mL} / \mathrm{min} /$ $\left.1.73 \mathrm{~m}^{2} ; \mathrm{p}<0.0001\right)$. As shown in Figure 1, they also had a greater age-adjusted prevalence of individuals with CKD (i.e., eGFR $<60 \mathrm{~mL} / \mathrm{min} / 1.73 \mathrm{~m}^{2}$ ). Moreover, as expected, there was a much higher proportion of individuals with increased serum anti-TPO $(43.9 \%$ vs. $15.8 \%, p<0.0001)$ or anti-TG antibodies $(33.7 \%$ vs. $11.3 \%, p<0.0001)$ in the hypothyroid subgroup. Similarly, compared with participants with no thyroid autoimmunity $(n=702)$, those with increased levels of anti-TPO and/or anti-TG $(n=213)$ had a greater ageadjusted prevalence of individuals with CKD (Figure 1).

Figure 2 shows the joint effects of TSH and thyroid autoimmunity on age-, and gender-adjusted values of eGFR. In this analysis, we found that a serum TSH $>4.0 \mathrm{mUl} / \mathrm{L}$ and increased titers of anti-thyroid antibodies exerted an additive effect on mean eGFR values (Figure 2). However, decreasing eGFR values appeared to be more strongly related with the increase in TSH, rather than with thyroid autoimmunity $(p<0.001$ for TSH, $p=0.12$ for thyroid autoimmunity, and $p=0.62$ for the interaction term). Essentially identical results were found when eGFR was included as categorical measure (eGFR $\leq 60$ vs. $>60 \mathrm{~mL} / \mathrm{min} /$ $1.73 \mathrm{~m}^{2}$ ) (data not shown).

\section{Discussion}

From a clinical practice viewpoint, it should be mentioned that clinically overt thyroid dysfunction, especially overt hypothyroidism, is accompanied by significant changes in renal blood flow, glomerular and tubular function, and electrolyte and water

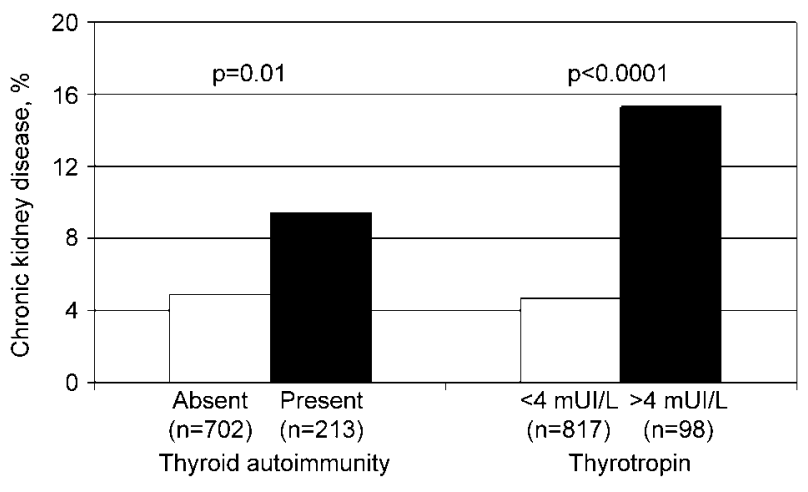

Figure 1 Age-adjusted prevalence of chronic kidney disease, as defined as estimated glomerular filtration rate $<60 \mathrm{~mL} / \mathrm{min} / 1.73 \mathrm{~m}^{2}$, by concentrations of serum thyrotropin and anti-thyroid antibodies.

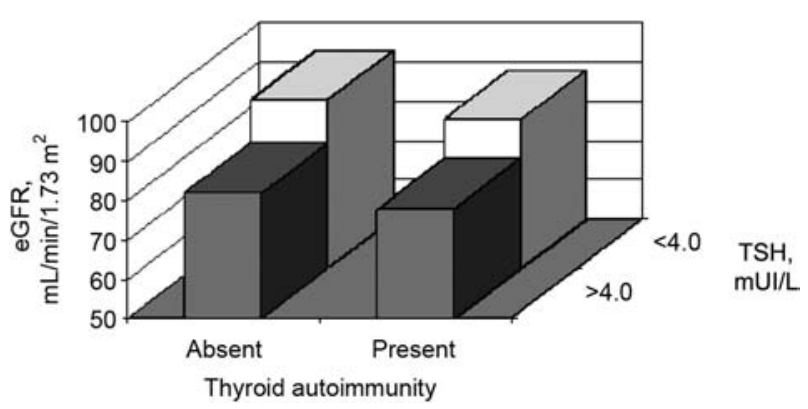

Figure 2 Joint effects of increased serum thyrotropin (TSH) concentrations and thyroid autoimmunity (defined as elevated anti-TPO and/or anti-TG antibodies) on estimated glomerular filtration rate values $(n=915)$.

Data are adjusted for age and gender. 
homeostasis. Conversely, a marked decline in kidney function is accompanied by changes in the synthesis, secretion, metabolism, and elimination of thyroid hormones $(1,2)$.

To our knowledge, at present there is no information available regarding the epidemiology of thyroid autoimmunity in individuals, including large non-US cohort with different levels of eGFR.

Lo et al. (4) recently reported that the prevalence of subclinical and clinical hypothyroidism in US adults was $\sim 24 \%$ among persons with an eGFR $<60$ $\mathrm{mL} / \mathrm{min} / 1.73 \mathrm{~m}^{2}$. Their study differed from ours in that their multi-ethnic US cohort had a greater prevalence of males, only $56 \%$ of hypothyroid cases were considered subclinical, and serum FT4 concentrations were not measured. Moreover, no information was available on the prevalence of thyroid autoimmunity in those with CKD.

In a recent study (5), we demonstrated in a large sample of unselected outpatients who presented to our laboratory between December 2005 and December 2007, the prevalence of subclinical primary hypothyroidism was $\sim 20 \%$ among persons with CKD. However, since no information was available on serum anti-thyroid antibodies in this study (5), we were unable to investigate the prevalence of thyroid autoimmunity and the effects of increased TSH concentrations and thyroid autoimmunity on eGFR values.

The major finding of our study is that there is a high frequency of thyroid autoimmunity $(34 \%)$ and subclinical primary hypothyroidism (26.4\%) in persons with an eGFR $<60 \mathrm{~mL} / \mathrm{min} / 1.73 \mathrm{~m}^{2}$. Notably, this finding was observed in a cohort of adult outpatients, and did not include subjects with abnormal serum FT4 concentrations or with CKD requiring chronic dialysis. The evaluation of subjects with such complications would almost certainly have confounded interpretation of the data. For example, it is known that in patients with renal failure it is not unusual to observe the low triiodothyronine (T3) syndrome; a common condition characterized by alterations in thyroid function likely due to non-thyroidal illness causes (11). In patients with renal failure, low serum free T3 concentrations are independently associated with increased risk of all-cause mortality $(12,13)$. Finally, as expected $(10,14)$, we also found that participants with lower eGFR were significantly older than those with normal or near-normal kidney function. Moreover, they also tended to have higher triglycerides and lower HDLcholesterol concentrations. However, fasting glucose and LDL-cholesterol concentrations did not differ significantly among eGFR categories. We believe that the lack of information on the use of lipid-lowering drugs did not allow us to observe a stronger statistical association between atherogenic dyslipidemia (i.e., high triglycerides and low HDL-cholesterol) and decreased kidney function. It is known that CKD patients have a greater prevalence of atherogenic dyslipidemia than those without CKD $(14,15)$.

Our study has several important limitations that merit comment. First, the cross-sectional design of the study precludes the establishment of causal or temporal relationships between thyroid autoimmunity, subclinical hypothyroidism and kidney disease. In particular, although our results were adjusted for age, it is known that the prevalence of CKD, thyroid autoimmunity and TSH concentrations increase markedly with age $(14,16)$. Thus, future prospective studies are needed to clarify these issues. Second, since our analysis depended on the use of automated databases to establish the presence of subclinical primary hypothyroidism and kidney disease, it may have led to some misclassification. In particular, we did not have any information on the causes of kidney disease, coexisting medical conditions and current use of medications, including thyroid replacement therapy or anti-thyroid drugs. In addition, although we evaluated the most common cause of subclinical primary hypothyroidism (i.e., chronic autoimmune thyroiditis, typically characterized by increased levels of anti-thyroid antibodies), no information was available on other less common causes of transient or permanent primary hypothyroidism, such as drug-induced hypothyroidism, subacute thyroiditis, radiation thyroiditis, or post-partum thyroiditis (9). However, we believe that the lack of these data does not detract from the novel and interesting finding of the high prevalence of thyroid autoimmunity among persons with reduced eGFR. Third, thyroid function tests could be requested when there was a clinical suspicion of altered thyroid function, thus tending to inflate the magnitude of the relationship. However, we excluded all patients with low or high serum FT4 levels, who are likely to have clinical symptoms of hypothyroidism or hyperthyroidism, respectively. Finally, we used an eGFR instead of a directly measured GFR to define CKD. It is known that current GFR equations have limited precision and systematically underestimate measured GFR at higher values (i.e., in populations without known CKD). However, current GFR equations facilitate the detection, evaluation, and management of CKD, and many organizations recommend the use of equations that estimate GFR for the evaluation of kidney function in large epidemiological studies and in clinical practice (10).

Despite these limitations, this analysis has several important strengths. First, as reported above, we excluded patients who required chronic dialysis and those who had overt thyroid dysfunction. Second, our clinical laboratory used uniform methods to collect data on thyroid function test results. Third, the availability of complete data on important risk factors, including age, gender, plasma glucose and the lipid profile, allowed us to provide an unbiased estimate for the relationship between subclinical hypothyroidism, thyroid autoimmunity and kidney disease. Finally, we evaluated a large sample, and found a strong, graded, relationship between eGFR and thyroid function tests, even within the reference intervals.

In conclusion, our findings suggest that there is a high prevalence of thyroid autoimmunity and subclinical primary hypothyroidism in a large non-US outpatient cohort of persons with CKD not requiring 
chronic dialysis. Further experimental and prospective studies are needed to confirm these findings and to elucidate the underlying biologic mechanisms before causality can be firmly established.

\section{Conflict of interest statement}

We declare that we have not accepted any funding or support from an organization that may in any way gain or lose financially from the results of our study, that we are not employed by an organization that may in any way gain or lose financially from the results of our study, and that we do not have any other conflicting interests.

\section{References}

1. Iglesias $P$, Diez JJ. Thyroid dysfunction and kidney disease. Eur J Endocrinol 2009;160:503-15.

2. Lim VS. Thyroid function in patients with chronic renal failure. Am J Kidney Dis 2001;38(4 Suppl 1):S80-4.

3. Hegedus L, Andersen JR, Poulsen LR, Perrild H, Holm B, Gundtoft $E$, et al. Thyroid gland volume and serum concentrations of thyroid hormones in chronic renal failure. Nephron 1985;40:171-4.

4. Lo JC, Chertow GM, Go AS, Hsu CY. Increased prevalence of subclinical and clinical hypothyroidism in persons with chronic kidney disease. Kidney Int 2005;67:1047-52.

5. Chonchol M, Lippi G, Salvagno G, Zoppini G, Muggeo M, Targher G. Prevalence of subclinical hypothyroidism in patients with chronic kidney disease. Clin J Am Soc Nephrol 2008;3:1296-300.

6. Walsh JP, Bremner AP, Bulsara MK, O'Leary P, Leedman PJ, Feddema $P$, et al. Subclinical thyroid dysfunction as a risk factor for cardiovascular disease. Arch Intern Med 2005;165:2467-72.
7. Rodondi N, Newman AB, Vittinghoff E, de Rekeneire N, Satterfield S, Harris TB, et al. Subclinical hypothyroidism and the risk of heart failure, other cardiovascular events, and death. Arch Intern Med 2007;165:2460-6.

8. lervasi G, Molinaro S, Landi P, Taddei MC, Galli E, Mariani $F$, et al. Association between increased mortality and mild thyroid dysfunction in cardiac patients. Arch Intern Med 2007;167:1526-32.

9. Surks MI, Ortiz E, Daniels GH, Sawin CT, Col NF, Cobin $\mathrm{RH}$, et al. Subclinical thyroid disease: scientific review and guidelines for diagnosis and management. J Am Med Ass 2004;291:228-38.

10. Stevens LA, Coresh J, Greene T, Levey AS. Assessing kidney function-measured and estimated glomerular filtration rate. N Engl J Med 2006;354:2473-83.

11. Adler SM, Wartofsky L. The non-thyroidal illness syndrome. Endocrinol Metab Clin North Am 2007;36:65772.

12. Zoccali C, Mallamaci F, Tripepi G, Cutrupi S, Pizzini P. Low triiodothyronine and survival in end-stage renal disease. Kidney Int 2006;70:523-8.

13. Enia G, Panuccio V, Cutrupi S, Pizzini P, Tripepi G, Mallamaci $F$, et al. Subclinical hypothyroidism is linked to micro-inflammation and predicts death in continuous ambulatory peritoneal dialysis. Nephrol Dial Transplant 2007;22:538-44.

14. National Kidney Foundation. K/DOQI clinical practice guidelines for chronic kidney disease: evaluation, classification and stratification. Am J Kidney Dis 2002;39 (Suppl 1):S1-266.

15. Chonchol M, Lippi G, Montagnana M, Muggeo M, Targher G. Association of inflammation with anaemia in patients with chronic kidney disease not requiring chronic dialysis. Nephrol Dial Transplant 2008;23:2879-83.

16. Hollowell JG, Staehling NW, Flanders WD, Hannon WH, Gunter EW, Spencer CA, et al. Serum TSH, T(4), and thyroid antibodies in the United States population (1988 to 1994): National Health and Nutrition Examination Survey (NHANES III). J Clin Endocrinol Metab 2002;87:489-99. 